\title{
Auditoria no âmbito da Atenção Primária: uma revisão integrativa da literatura
}

\author{
Adson Bruno Rodrigues de MENESES ${ }^{1}$ \\ Aydwlha Moniq Barbosa de SANTANA ${ }^{2}$ \\ Felipe Artur Gomes de ASSIS ${ }^{3}$ \\ Waleska de Brito NUNES \\ Luciana Dantas Farias de ANDRADE
}

\author{
${ }^{1}$ Acadêmico de Enfermagem pela UFCG, ads0n_brun0@hotmail.com \\ ${ }^{2}$ Acadêmica de Enfermagem pela UFCG, aydwlha@hotmail.com \\ ${ }^{3}$ Acadêmico de Enfermagem pela UFCG, felipearturufcg@hotmail.com \\ ${ }^{4}$ Acadêmica de Enfermagem pela UFCG, waleskinhabio@hotmail.com \\ ${ }^{5}$ Orientadora. Professora do Curso de Graduação em Enfermagem da UFCG - Campus Cuité. Paraíba. Mestre em \\ Enfermagem. luciana_dantas_farias@yahoo.com.br
}

Recebido em: 14/09/2012 - Aprovado em: 22/12/2012 - Disponibilizado em: 30/12/2012

\begin{abstract}
Resumo:
A auditoria na atenção primária surgiu para controlar o próprio sistema público de saúde e com isso regulando os preceitos constitucionais e normativos do SUS. O presente estudo teve como objetivo realizar uma revisão integrativa da literatura sobre auditoria no âmbito da atenção primária. A principal vantagem deste tipo de estudo reside no fato de permitir investigar uma ampla gama de fenômenos por meio de pesquisa em materiais já elaborados, possibilitando o aprimoramento de idéias e conceitos. Quanto à seleção dos artigos, selecionaram dez estudos e um trabalho de conclusão de curso de especialização. No entanto, apenas três explicitam claramente a auditoria na atenção primária juntamente com trabalho de conclusão de curso de especialização, enquanto os demais expõem a temática de forma indireta. É importante que seja feito um acompanhamento da qualidade da assistência à saúde na atenção Primária, tendo em vista que suas ações poderão implicar em melhores ou piores resultados.
\end{abstract}

Palavras-Chave: Auditoria em Enfermagem. Atenção Primária. Saúde. Enfermagem. Saúde Pública.

\section{Audit under Primary Care: an integrative literature review}

\begin{abstract}
:
The audit in Primary emerged to control their own public health system and thereby regulating the constitutional precepts and regulations of SUS. The present study aimed to conduct an integrative review of the literature on audit within Primary Care. The main advantage of this type of study lies in the fact allow to investigate a wide range of phenomena through research on materials already developed, enabling the enhancement of ideas and concepts. Regarding the selection of articles selected ten studies and a conclusion work specialization course. However, only three clearly explain the audit in Primary along with work completion for specialization, while others expose the issue indirectly. It is important that it is done to monitor the quality of health care in primary care, considering that their actions may result in better or worse results.
\end{abstract}

Keywords: Nursing Audit. Primary Care. Health. Nursing. Public Health

\section{INTRODUÇÃO}

A prestação de serviços de saúde pode ser compreendida como uma estrutura que une o avanço tecnológico o conhecimento técnico cientifico dos profissionais de saúde, bem como os aperfeiçoamentos desses profissionais. Esse conjunto que integram essa estrutura de prestação de serviços visa atender as necessidades dos usuários com qualidade $^{1}$. De acordo com a evolução da doença, podemos citar os três níveis da assistência em saúde como: atenção primária à saúde, atenção secundária à saúde e atenção terciária à saúde. 
Segundo o Manual para Organização da atenção básica ${ }^{2}$, a atenção básica é um conjunto de ações, de caráter individual ou coletivo, situadas no primeiro nível de atenção nos sistemas de saúde, voltada para a promoção de saúde, prevenção de agravos, o tratamento e a reabilitação. Apresentada na literatura internacional como atenção primária à saúde (APS).

Diante disto, Ministério da Saúde $^{3}$ destaca que a atenção primária à saúde se apresenta como estratégia organizativa da atenção à saúde definida pelos princípios e diretrizes que esses serviços devem proporcionar e que devem incluir: uma atenção generalizada sem restrição a faixas etárias, gêneros ou problemas de saúde; acesso integral e contínuo com trabalho em equipe; atenção centrada na pessoa e não na doença; atenção dirigida à família e a comunidade de modo coordenado incluindo o acompanhamento do usuário nos outros níveis de atenção e fornecer apoio constante aos usuários nos aspectos relacionados à saúde e bem estar.

Deste modo, atenção primária atende uma grande demanda, correspondendo a elevadas consultas médicas e de enfermagem, sendo essa atenção a porta de entrada para o sistema de atenção à saúde no Brasil. Além desse papel, a atenção primária também é responsável por articular as demandas sanitárias, como a vigilância e controle de doenças, bem como riscos de adoecimento, além da educação em saúde. Portanto esse nível de atenção utiliza a tecnologia leve e leve dura que envolve um conjunto de saberes e complexa integração com ações individuais e coletivas, curativas e preventivas, assistenciais e educativas ${ }^{4}$.

Como em qualquer instituição prestadora de serviços, na atenção primária deve haver uma forma de auditoria visando à avaliação do serviço prestado. Por sua vez, a auditoria na atenção primária, em um sistema municipal de saúde é uma tarefa complexa, pois a atenção primária não deve ser encarada como um componente isolado de um dado sistema, e são inúmeros os fatores responsáveis pelo seu maior ou menor desempenho.

O Sistema Nacional de Auditoria foi instituído pela lei no 8689/93 e compete a esse a avaliação técnico-científica, contábil, financeira e patrimonial do SUS, sendo realizada de forma descentralizada. ${ }^{5}$

A auditoria na atenção primária surgiu para controlar o próprio sistema público de saúde e com isso regulando os preceitos constitucionais e normativos do SUS. ${ }^{5}$

Para tanto é importante ressaltar que auditoria pode ser considerada um elemento essencial para mensurar a qualidade da assistência, oferecendo subsídios aos profissionais para (re) orientar suas 
atividades, estimulando a reflexão individual e coletiva e nortear o processo de educação permanente $^{6}$.

Portanto a auditória propõe avaliar a qualidade da assistência prestada ao usuário do serviço de saúde, propondo soluções para que essa assistência prestada tenha uma melhor qualidade. Diante dessa assertiva, a qualidade da assistência da atenção primária de Saúde pode ser avaliada por diversos parâmetros.

Deste modo, partindo da necessidade de publicações sobre auditoria no âmbito da atenção primária, observou-se o interesse de acadêmicos pela temática através de uma disciplina curricular do curso de Bacharelado em Enfermagem intitulada "Auditoria em Enfermagem". Visualizando a auditoria como um dos instrumentos para avaliar a qualidade do cuidar, este estudo tem como objetivo realizar uma revisão integrativa da literatura sobre auditoria no âmbito da atenção primária.

\section{METODOLOGIA}

Para atingir o objetivo proposto foi realizada uma revisão integrativa da literatura. A principal vantagem deste tipo de estudo reside no fato de permitir investigar uma ampla gama de fenômenos por meio de pesquisa em materiais já elaborados, possibilitando o aprimoramento de idéias e conceitos.

A revisão integrativa segundo Silva, Souza e Carvalho ${ }^{7}$, determina o conhecimento atual sobre uma temática específica, já que é conduzida de modo a identificar, analisar e sintetizar resultados de estudos independentes sobre o mesmo assunto, contribuindo, pois, para uma possível repercussão benéfica ao tema estudado.

Sabemos que a escolha do tema é de importância na elaboração do projeto e requer bastante habilidade dos pesquisadores. $\mathrm{Na}$ escolha do tema os pesquisadores consideraram o interesse próprio e aptidão na área pesquisada, além de escasso material publicado.

Este estudo baseou-se na pesquisa de artigos publicados em periódicos científicos nacionais e bibliotecas eletrônicas disponíveis na Scientific Electronic Library Online (Scielo), e (Dedalus), que apresentam periódicos nacionais com artigos pertinentes ao tema, sendo eles: Revista Brasileira de Enfermagem, Revista Nursing, Revista Eletrônica de Enfermagem, Acta Paulista de Enfermagem.

O estudo foi desenvolvido na Universidade Federal de Campina Grande (UFCG)- Centro de Educação e Saúde (CES), situada na cidade de Cuité, no Curimataú paraibano, através da disciplina Auditoria em 
Enfermagem, oferecida na Matriz curricular do $8^{\circ}$ período do Curso de Bacharelado em Enfermagem.

É importante destacar que os pesquisadores levaram em consideração as observâncias éticas contempladas no Código de Ética dos Profissionais de Enfermagem Resolução 311/2007 do Conselho Federal de Enfermagem, no que se refere à elaboração de trabalhos científicos, como mostra os artigos a seguir:

1. “Art. 88 - Ter reconhecida sua autoria ou participação em produção técnico-científica." RESPONSABILIDADES E DEVERES

2. “[..]. Art. 91 - Respeitar os princípios da honestidade e fidedignidade, bem como os direitos autorais no processo de pesquisa, especialmente na divulgação dos seus resultados.

3. Art. 92 - Disponibilizar os resultados de pesquisa à comunidade científica e sociedade em geral.

4. Art. 93 - Promover a defesa e o respeito aos princípios éticos e legais da profissão no ensino, na pesquisa e produções técnico-científicas."

\section{RESULTADOS E DISCUSSÃO}

Quanto à seleção dos artigos, os autores selecionaram dez estudos e um trabalho de conclusão de curso de especialização. No entanto, dos dez artigos apenas três explicitam claramente a auditoria na atenção primária juntamente com trabalho de conclusão de curso de especialização, enquanto os demais expõem a temática de forma indireta.

Para tanto, é importante enfatizar que desses três, o titulo não fala claramente sobre auditoria na atenção primária e sim de avaliação da atenção básica, seja através de prontuário; dos registros de crescimento e desenvolvimento; avaliação de atendimento como pré- natal; avaliação de estrutura física; bem como avaliação do atendimento nas consultas médicas e de enfermagem.

Costa $^{1}$ citam que as atividades de auditoria na atenção primária geralmente realizam as seguintes ações: acompanhamento nas unidades básicas de saúde, averiguação de denuncias de irregularidades, a efetivação do cronograma de atividades e presenças dos profissionais de saúde com sua respectiva carga horária, bem como a qualidade da assistência prestada. Essas ações de auditorias visam a democratização dos direitos dos usuários, pois o direito a saúde é dever do estado e direitos de todos que esta na constituição de 1988.

Para facilitar as ações de auditoria o DENASUS em conjunto com a Coordenação da Atenção Primária do Ministério da Saúde formularam um roteiro de auditoria operativa em Unidade Básica de Saúde. Os roteiros 
foram organizados dentro do esquema clássico de avaliação com a abordagem de "Estrutura, Processo e Resultado", que juntos, abordam as unidades básicas de saúde desde as condições físicas até as mudanças na assistência primária, na população atendida, verificadas em seus resultados.

As avaliações da auditoria em atenção primária são utilizadas de forma contínua sobre estruturas organizacionais e funcionais dos serviços de saúde para calcular a eficiência das ações e atividades de saúde. Cujos resultados são apresentados em forma de relatórios à administração do serviço do sistema de saúde. Nesse relatório deve conter "ações burocráticas funcionais da produção de serviços e controle do equilíbrio financeiro, os auditores executam em seus planos, a avaliação do desempenho na rede de serviço $[\ldots]^{1}$

Já o trabalho de conclusão de curso de especialização utiliza em seu titulo a palavra auditoria dando ênfase à auditoria na atenção primaria à saúde, destacando a solicitação de exames complementares para essa avaliação.

Deste modo, ver-se a grande necessidade de publicações falando sobre a auditoria na atenção primária. Além a utilização do termo auditoria para facilitar a busca de interessados no assunto.

Além do mais, é importante destacar que dos estudos publicados e selecionados para a pesquisa, muitos não enfatizam em seu estudo a avaliação (auditoria) e $\operatorname{sim}$ a destacam alguns mecanismos de funcionamento da atenção primária.

Enquanto que o trabalho de conclusão de curso de especialização destaca em seu estudo a auditoria, como ocorre essa auditoria na atenção primária a partir de solicitação de exames complementares.

Todos os estudos, ao falar diretamente na auditoria, evidenciaram o prontuário do paciente como ferramenta fundamental para a auditoria e destacaram que as anotações nele presentes, demonstram diretamente a qualidade da assistência prestada, tendo em vista que no prontuário devem ser anotadas todas as intervenções realizadas.

\section{CONSIDERAÇÕES FINAIS}

É de suma importância que seja feito um acompanhamento da qualidade da assistência à saúde na atenção primária, tendo em vista que suas ações poderão implicar em melhores ou piores resultados nos outros níveis de atenção à saúde. Quando dentro das unidades básicas de saúde, atuam profissionais comprometidos com as ações de prevenção e promoção da saúde, certamente diminui-se o número de pacientes necessitando das assistências, secundária e terciária e consequentemente a melhoria da qualidade de vida dos usuários da saúde. 
Isso porque se o serviço da atenção primária estiver sendo efetivo em realizar as atividades educativas, consultas necessárias com a equipe multiprofissional e registrando todos os resultados, fica bem mais fácil fazer o rastreamento das doenças crônicas e suas complicações e ainda das necessidades de cada comunidade cadastrada nas ESF específicas.

A qualidade dos registros reflete diretamente na avaliação do serviço quando se faz uma auditoria, pois, o instrumento principal do auditor, são as anotações registradas em prontuários, e em contrapartida, é urgente a sensibilização de cada profissional envolvido na assistência para que sejam fielmente anotadas todas as ações implementadas durante a assistência prestada nas UBS's.

Por fim fica claro que na atenção primária à saúde, deve-se atentar para o sistema de auditorias que abordam diversos aspectos da assistência, não apenas o de materiais e insumos utilizados. Estudos que analisem esse aspecto amplo da auditoria em saúde na atenção primária, tornam-se necessários até para estimular nos profissionais da saúde, melhores rotinas de trabalho nas UBS e por conseguinte, uma melhor assistência direcionada para integralidade como propõe o SUS.

\section{REFERENCIAS:}

1- Costa MS, Forte BP, Alves MDS, Viana JF, Oriá MOB. Revista Brasileira Enfermagem, Brasília (DF) 2004 jul-ago;57(4):497-9.

2- Orientações para proceder auditoria. Manual para Organização da Atenção Básica. Ministério da Saúde Departamento Nacional de Auditoria do SUS DENASUS, Brasília, 1998

3- Ministério da Saúde, Orientações para proceder auditoria. Manual para Organização da Atenção Básica. Ministério da Saúde Departamento Nacional de Auditoria do SUS DENASUS, Brasília, 2004

4- SALA A; NEMES M I B \& COHEN D D . Metodologia de avaliação do trabalho na atenção primária à saúde. Cad. Saúde Pública, Rio de Janeiro, (1998)14 (4):741-751, out-dez.

5- Hering FG, Auditoria em saúde: Avaliação da atenção primária em saúde através da solicitação de exames complementares no município de Gravataí, Porto Alegre, 2008

6- Faraco MM, Albuquerque GL. Revista Brasileira Enfermagem, Brasília (DF) 2004 jul-ago; 57(4):421-4.

7- Souza MT, Silva MD, Carvalho R. Revisão integrativa: o que é e como fazer. Einstein. 2010; 8(1 Pt 1):1026

8- Conselho Federal De Enfermagem, COFEN. Resolução 311, 2009. Disponível em: http://site.portalcofen.gov.br/node/4 345. 